\title{
Suppression of scinderin modulates epithelial-mesenchymal transition markers in highly metastatic gastric cancer cell line SGC-7901
}

\author{
XIAO-MIN CHEN ${ }^{1}$, JUN-MING GUO ${ }^{1}$, PING CHEN $^{2}$, LIAN-GANG MAO ${ }^{2}$, \\ WEI-YUN FENG ${ }^{2}$, DONG-HAI LE ${ }^{2}$ and KE-QIANG LI ${ }^{1,2}$ \\ ${ }^{1}$ Ningbo University School of Medicine, Ningbo, Zhejiang 315211; \\ ${ }^{2}$ Oncomolecularbiology Laboratory of Ningbo No. 2 Hospital, Ningbo, Zhejiang 315010, P.R. China
}

Received November 30, 2013; Accepted June 17, 2014

DOI: $10.3892 / \mathrm{mmr} .2014 .2523$

\begin{abstract}
Scinderin is a $\mathrm{Ca}^{2+}$-dependent filamentous actin (F-actin) severing and capping protein, which has a key role in regulated secretion. However, little is known regarding the function and mechanism of scinderin in human carcinoma development and progression. In the present study, the biological function of scinderin was investigated using a cell proliferation assay, flow cytometric analysis and a Transwell assay in highly tumorigenic and the metastatic human gastric cancer cell line SGC-7901 transfected with scinderin-small hairpin RNA lentivirus. The changes in the expression of epithelial-mesenchymal transition (EMT) markers were also investigated. The results indicated that scinderin knockdown effectively suppressed proliferation, reduced migration and arrested the cell cycle of the SGC-7901 cells at G2/M phase. Furthermore, scinderin knockdown altered the expression of EMT markers; the expression of E-cadherin was significantly upregulated, along with an evident decrease in N-cadherin and $\beta$-catenin expression. In conclusion, the present study suggested that suppression of scinderin impaired proliferation and migration of gastric cancer SGC-7901 cells and attenuates its EMT process. Scinderin may therefore be a potential target for tumor EMT and therapy against gastric cancer.
\end{abstract}

Correspondence to: Dr Ke-Qiang Li or Mr. Dong-Hai Le, Oncomolecularbiology Laboratory of Ningbo No. 2 Hospital, 41 Xibei Road, Ningbo, Zhejiang 315010, P.R. China

E-mail: chasejxmc@163.com

E-mail: dhyue@foxmail.com

Abbreviations: F-actin, filamentous actin; EMT, epithelial-mesenchymal transition; RTCA, real-time cell analyzer; RT-qPCR, real-time quantitative polymerase chain reaction

Key words: scinderin, gastric cancer, epithelial-mesenchymal transition, metastasis

\section{Introduction}

Scinderin (or adseverin), a member of the gelsolin superfamily, is a $\mathrm{Ca}^{2+}$-dependent filamentous actin (F-actin) severing and capping protein with three actin-, two $\mathrm{PIP}_{2}$ - and two $\mathrm{Ca}^{2+}$-binding sites (1-4). Scinderin is expressed largely in endocrine tissues and secretory cells. During human development, scinderin expression is different from that in adults: It is highly expressed in the human fetal kidney, very weakly in the brain and intestine but nowhere else; in human adult tissues, scinderin exhibits strong expression in the kidney and low expression in the heart; however, no expression is observed in the adrenal gland (5).

It has been demonstrated that scinderin has important roles in exocytosis, megakaryopoiesis, autoimmune disorders and tumorigenesis. Scinderin is known to regulate translocation of secretory vesicles by controlling F-actin dynamics (disassembly $\leftrightarrow$ assembly) during secretion (6-8). Overexpression of scinderin on human airway epithelial cells may inhibit mucin secretion (9). Scinderin has also been found in platelets. Previous studies have suggested that the expression of scinderin in the megakaryoblastic cell line MEG-01 induces cell differentiation, polyploidization, maturation and apoptosis with release of plateletlike particles, while cell proliferation and tumorigenesis in nude mice are inhibited (10). It has been reported that single-nucleotide polymorphisms (SNPs) in scinderin have a prominent effect in multiple sclerosis (11). Overexpression of scinderin triggers human non-small-cell lung carcinoma cell line IGR-Heu resistance to cytolytic T lymphocytes (12). Furthermore, scinderin is capable of preventing mitochondria-mediated apoptosis by directly binding to voltage-dependent anion channels in ciplatin-resistant cells (13).

Gastric cancer is one of the most common primary gastrointestinal cancers in the world. Despite improvements in diagnostic modalities, outcomes for patients with gastric cancer remain extremely poor, with the majority of cancer patients dying from the occurrence of metastases rather than their primary tumors. Due to its complexity, the process of tumor metastasis remains poorly understood. Epithelial-mesenchymal transition (EMT) is a transdifferentiation process during which epithelial cells lose their epithelial polarity and cell-cell contacts, gain mesenchymal 
properties, and become motile and invasive $(14,15)$. In this process, fundamental changes in gene expression are involved in the disruption of epithelial polarity (e.g., E-cadherin and cytokeratins) and establishment of a mesenchymal phenotype (e.g. $\mathrm{N}$-cadherin and vimentin) (16). EMT is physiologically relevant during embryogenesis and in adult tissue for wound healing. However, EMT is also associated with tumor metastasis. Of note, the majority of solid carcinoma cells (including breast, prostate, pancreatic, colon and gastric cancer, etc.) have been reported to undergo either partial or complete EMT so as to become motile and invasive. Currently, EMT is regarded as an important mechanism of tumor metastasis, having crucial roles in the early process of therioma metastases (15). Therefore, it is essential to find molecules involved in both the positive and negative regulation of EMT. It has been demonstrated that signaling pathways, including TGF- $\beta$, Wnt/ $\beta$-catenin, Notch, Hedgehog, interleukin-6/signal transducer and activator of transcription 3 and nuclear factor- $\kappa \mathrm{B}$ trigger EMT by inducing Snail1, Snail2, Twist1, Twist2, zinc finger E-box binding homeobox (ZEB)1 and ZEB2 expression (17-19). Furthermore, non-coding RNAs (including microRNAs and long non-coding RNAs) also have critical roles in the regulation of the EMT (20-22).

Although it has been demonstrated that scinderin expression is low or absent in the human adult stomach, no studies, to the best of our knowledge, have reported the biological effect of scinderin on human gastric cancer. In the present study, the effects of scinderin knockdown on cell cycle, proliferation and migration of the highly tumorigenic and metastatic human gastric cancer cell line SGC-7901 (scinderin is highly expressed) were investigated. Furthermore, the changes in E-cadherin, $\mathrm{N}$-cadherin and $\beta$-catenin expression following scinderin knockdown were examined and the role of scinderin in the tumor EMT process was analyzed.

\section{Materials and methods}

SGC-7901 cell line. The human gastric cancer cell line SGC-7901 was purchased from Chinese Academy of Sciences (Shanghai, China). The cells were cultured in RPMI-1640 medium (Invitrogen Life Technologies, Carlsbad, CA, USA) with $10 \%$ fetal bovine serum (FBS) in a humidified incubator containing $5 \% \mathrm{CO}_{2}$ at $37^{\circ} \mathrm{C}$.

Construction of scinderin-small hairpin (sh)RNA lentiviral plasmid. The target shRNA against the scinderin gene (Gene Bank accession NM_033128) was designed as follows: 5'-CGA GAT GAG CTG ACA ACA T-3'. Oligonucleotides encoding shRNA sequences were synthesized (Genechem Corporation, Shanghai, China) and annealed into double strands. Double-stranded DNAs were inserted into HpaI/XhoI restriction sites of lentiviral frame plasmid (Genechem Corporation), encoding green fluorescent protein (GFP). They were then transfected into Escherichia coli and positive recombinant lentiviral plasmids were selected by polymerase chain reaction (PCR), using the primers 5'-GCC CCG GTT AAT TTG CAT AT-3' and 5'-GAG GCC AGA TCT TGG GTG-3'. The conditions for PCR were denaturation at $94^{\circ} \mathrm{C}$ for $30 \mathrm{sec}$, then $94^{\circ} \mathrm{C}$ for $30 \mathrm{sec}, 55^{\circ} \mathrm{C}$ for $30 \mathrm{sec}$ and $72^{\circ} \mathrm{C}$ for $30 \mathrm{sec}$, for 30 cycles and extension at $72^{\circ} \mathrm{C}$ for $6 \mathrm{~min}$. The products were then verified by electrophoretic analysis on a $1.5 \%$ agarose gel containing ethidium bromide and DNA sequencing. The lentiviral vectors expressing GFP alone were used as the control.

Packaging and titration of lentiviral vectors. The recombinant lentiviral plasmid was co-transfected into 293T cells with packaging plasmids (pHelper 1.0 including gag/pol and pHelper 2.0 including VSVG; Genechem Corporation) by Lipofectamine 2000 (Invitrogen Life Technologies) to produce target lentivirus. Following $48 \mathrm{~h}$, the virus in the supernatant was collected and the virus titer was measured following the dilution method: i) a total of $4 \times 10^{4}$ cells/well of the 293T cells were seeded in a 96-well plate in Dulbecco's modified Eagle's medium (Gibco Life Technologies, Carlsbad, CA, USA) supplemented with $10 \%$ FBS and cultured overnight; ii) the serial diluted vectors were added to the cultured cells and continued to culture for $48 \mathrm{~h}$; iii) the GFP-positive cells were counted. The titer was represented as transduction unit (TU) per milliliter concentrated vector (TU/ml): Titer $=$ GFP-positive cell number/dilution $\times 10^{3}$.

Establishment of the stable scinderin-silenced cell line $S G C$-7901. A preliminary experiment identified that the best multiplicity of infection (MOI) of SGC-7901 cells was 10 (MOIs of 1, 10 and 20 were tested). Under these best transfection conditions, scinderin-shRNA lentivirus was transfected into the SGC-7901 cells. The efficiency of transfection was observed under a fluorescence microscope (MicroPublisher 3.3 RTV; Olympus, Tokyo, Japan). The effects of gene silencing were confirmed by quantitative (q)PCR and western blot analyses. The cells were divided into three groups: The CON group (uninfected), the NC group (transfected with negative control lentivirus) and the KD group (transfected with scinderin-shRNA lentivirus).

Cell migration assay. Cell migration was assessed using a Transwell assay (Corning Incorporated Life Sciences, Lowell, MA, USA). Transwell insert chambers were firstly rehydrated with $100 \mu \mathrm{l}$ serum-free medium at $37^{\circ} \mathrm{C}$ for $1 \mathrm{~h}$. Following removing the medium from the chambers, $1 \times 10^{5}$ cells in $100 \mu 1$ serum-free medium were added to the upper chamber, with $600 \mu \mathrm{l}$ medium containing 30\% FBS in the lower chamber, and then cultured for $24 \mathrm{~h}$. Subsequently, the cells were removed from the top side of the membrane using a cotton-tipped swab, and the invaded cells attached to the bottom of the membrane were fixed with $10 \%$ glutaraldehyde, stained with $0.1 \%$ crystal violet (Shanghai Genebase Gene-Tech Co., Ltd, Shanghai, China) and photographed under an inverted microscope (MicroPublisher 3.3 RTV; Olympus). Next, the cells were destained with $10 \%$ acetic acid, and the optical density (OD) of the solution was measured at $570 \mathrm{~nm}$. The OD value was directly proportional to the cell quantity and indirectly represented the cellular migration ability.

$R T-q P C R$. Total RNA was extracted from cells using TRIzol (Invitrogen Life Technologies), according to manufacturer's instructions. Quantitative RNA expression was measured by the SmartSpec ${ }^{\mathrm{TM}}$ Plus Spectrophotometer (Bio-Rad, Hercules, CA, USA). Reverse transcription in a $20 \mu \mathrm{l}$ system was performed using the GoTaq ${ }^{\circledR}$ 2-step RT-qPCR System kit (Promega Corporation, Madison, WI, USA) following the manufacturer's 
instructions. qPCR was performed using the MX3005P qPCR System (Stratagene, La Jolla, CA, USA). Primers for qPCR were scinderin, forward 5'-TCT GCG TTC CTG ACT GTT C-3' and reverse 5'-GAC CTC CTT TCT TTG ATG TTC C-3'; GAPDH, forward 5'-TGA CTT CAA CAG CGA CAC CCA-3' and reverse 5'-CAC CCT GTT GCT GTA GCC AAA-3'; E-cadherin, forward 5'-CCA TCG CTT ACA CCA TCC T-3' and reverse 5'-GCT GTT GCT GTT GTG CTT-3'; $N$-cadherin, forward 5'-CTC CTA TGA GTG GAA CAG GAA CG-3' and reverse 5'-TTG GAT CAA TGT CAT AAT CAA GTG CTG TA-3'; $\beta$-actin, forward 5'-TCG TGC GTG ACA TTA AGG-3' and reverse 5'-AAG GAA GGC TGG AAG AGT-3'. The relative mRNA expression was calculated with the $2^{-\Delta \Delta C T}$ method. $G A P D H$ or $\beta$-actin were used as normalizers for each sample. All of the experiments were repeated in biological duplicate.

Western blot analysis. The cells were lysed with radioimmunoprecipitation assay (RIPA) lysis buffer (Bioteke Corporation, Beijing, China), and the protein concentration was measured by bicinchoninic acid assay (BeyotimeCorporation,Nantong,China). The total cell proteins were separated by $10 \%$ SDS-PAGE and transferred to nitrocellulose filter (NC) membranes (Millipore Corporation, Bedford, MA, USA). The NC membranes were firstly blocked by $5 \%$ skimmed milk for $1 \mathrm{~h}$ and then incubated overnight at $4^{\circ} \mathrm{C}$ with primary antibodies, including scinderin rabbit mAb (1:1,000, Santa Cruz Biotechnology, Dallas, TX, USA), GAPDH mouse mAb (1:1,000, Santa Cruz Biotechnology), E-cadherin rabbit mAb (1:1,000, Cell Signaling Technology, Danvers, MA, USA), N-cadherin rabbit pAb (1:1,000, Cell Signaling Technology), $\beta$-catenin rabbit mAb $(1: 1,000$, Cell Signaling Technology) and $\beta$-actin rabbit mAb (1:1,000, Cell Signaling Technology). Thereafter, blots were stained with fluorescent secondary antibodies IRDye $800 \mathrm{CW}$ Goat anti-Rabbit IgG $(\mathrm{H}+\mathrm{L})(1: 10,000$, LI-COR Bioscience, Lincoln, NE, USA) or IRDye 800CW Goat anti-Mouse IgG (H+L) (1:10,000, LI-COR Bioscience) and protein bands were visualized using an Odyssey Imaging system (LI-COR Bioscience). GAPDH or $\beta$-actin was used as the internal control.

Real-time cell proliferation assay (RTCA). The real-time cell proliferation experiment was performed with a RTCA single plate (SP) instrument (Roche Applied Science, Mannheim, Germany), which was placed in a $37^{\circ} \mathrm{C}$ incubator with $5 \% \mathrm{CO}_{2}$. Based on the manufacturer's instructions, the background signal of the culture medium was first measured by adding $100 \mu \mathrm{l}$ medium to 96-well plates (E-plate 96, Roche Applied Science) containing gold microelectrodes on its bottom. Next, the cells were seeded in the special 96 -well plates at density of 8,000 cells/well. Following $30 \mathrm{~min}$ of incubation at room temperature, the cells were placed in the RTCA SP and monitored every $15 \mathrm{~min}$ for $160 \mathrm{~h}$. Data analysis was performed using RTCA software 1.2 supplied with the instrument. The RTCA software comprised the xCELLigence system, which converts impedance values into cell index $(\mathrm{CI})$ values corresponding to each well. The CI value is directly proportional to the quantity of cells and was used as an indirect measure for cellular proliferation capability.

Cell cycle analysis. SGC-7901 cells stably transfected with scinderin-shRNA or empty lentiviral vector were firstly cultured

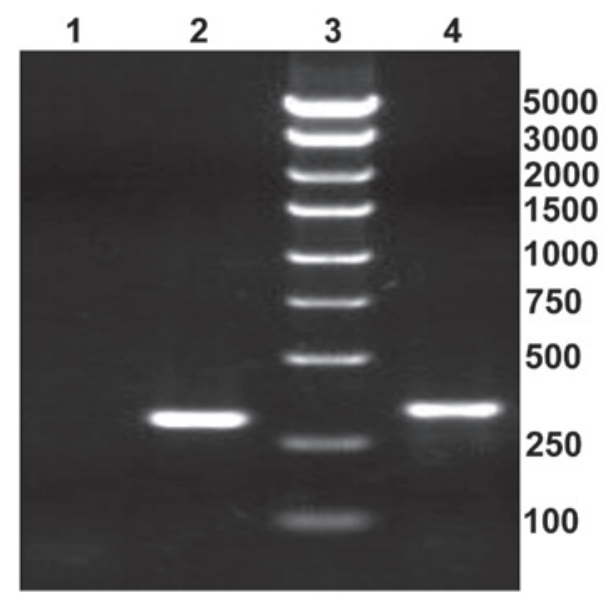

Figure 1. Electrophoresed polymerase chain reaction products of recombinant lentiviral plasmids. Lane $1, \mathrm{ddH}_{2} \mathrm{O}$; lane 2, non-shRNA lentiviral plasmids; lane 3, marker; lane 4, scinderin-shRNA recombinant lentiviral plasmids. shRNA, small hairpin RNA.

in serum-free medium for $24 \mathrm{~h}$ for synchronization in G0/G1 phase, and then cultured in RPMI-1640 medium with 10\% FBS for another $24 \mathrm{~h}$. The collected cells were washed twice with phosphate-buffered saline (PBS) and fixed in ice-cold $70 \%$ ethanol overnight at $-20^{\circ} \mathrm{C}$. Next, the cell pellets were washed twice with ice-cold PBS and stained with propidium iodide solution (BD Biosciences, San Jose, CA, USA) for $20 \mathrm{~min}$ in the dark. Finally, the cells were analyzed using the BD FACSCalibur Flow Cytometer (BD Biosciences) for their DNA content. The percentages of cells in the different phases of the cell cycle were determined with Cell Quest Pro software 3.1 (BD Biosciences).

Statistical analysis. Data are presented as the mean \pm standard deviation. The Student's t-test was used in quantitative data analysis, $\mathrm{P}<0.05$ was considered to indicate a statistically significant difference. All statistical analysis was performed with SPSS 20.0 software (SPSS, Inc., Chicago, IL, USA).

\section{Results}

Construction of scinderin-shRNA lentiviral plasmid. The double-stranded DNAs encoding shRNA sequences were inserted into $\mathrm{HpaI} / \mathrm{XhoI}$ restriction sites of the lentiviral frame plasmids. The electrophoresed PCR products of the recombinant lentiviral plasmids were as follows: The length of positive clones containing shRNA was 343 bp and the length of blank clones was 299 bp (Fig. 1). Furthermore, DNA sequencing demonstrated that the RNA interference sequence targeting scinderin was coincident with the anticipative results (data not shown). This suggested that the shRNA sequence targeting scinderin was successfully inserted into the lentiviral plasmid.

Packaging and titration of lentivirus. Recombinant lentivirus were produced in $293 \mathrm{~T}$ cells co-transfected with the recombinant lentiviral plasmid and packaging plasmids. The viral titer indicated that the recombinant lentivirus was packaged successfully and the viral titer was $\leq 6 \sim 8 \times 10^{8} \mathrm{TU} / \mathrm{ml}$ (Fig. 2).

Transfection efficiency of lentivirus in gastric cancer cell line SGC-7901. The preliminary experiment found that the optimal 
MOI of SGC-7901 cells was 10. Following transfection under these optimal conditions for $96 \mathrm{~h}$, the transfection efficiency was determined to be $>90 \%$ by counting GFP-positive cells under the fluorescent microscope. The result demonstrated that scinderin-shRNA lentivirus was transfected into SGC-7901 cells successfully.

Interference efficiency of scinderin in SGC-7901 cells. The silencing of scinderin was confirmed by qPCR and western blot analyses. The results demonstrated that following transfection into the SGC-7901 cells, both the mRNA and protein levels of scinderin were significantly decreased in the KD group compared with the CON and NC groups (Fig. 3). This suggested that stable scinderin-silenced cancer cells were established and appropriate for use in subsequent experiments.

Scinderin knockdown suppresses migration of SGC-7901 cells. The migration assay demonstrated that the number of cells penetrating the membrane of chambers was significantly lower in the KD group than that in the $\mathrm{NC}$ group $(\mathrm{P}<0.05$, Fig. 4). This result indicated that scinderin knockdown may attenuate migration of SGC-7901 cells.

Scinderin mediates EMT marker expression in SGC-7901 cells. Considering scinderin knockdown inhibits the migration of the metastatic human gastric cancer cell line SGC-7901, it was investigated whether scinderin mediates EMT, a critical event in tumor metastases. qPCR and western blot analyses revealed that concomitant with an evident decrease in $\mathrm{N}$-cadherin expression, E-cadherin expression in the KD group was significantly upregulated compared with that in the NC group (Fig. 5). Furthermore, it was observed that scinderin knockdown decreased the expression of $\beta$-catenin protein (Fig. 5B), an important regulatory molecule in EMT. Therefore, these results illustrated that scinderin knockdown may inhibit the EMT process in SGC-7901 cells.

Scinderin knockdown suppresses proliferation and arrests cell cycle of SGC-7901 cells. Furthermore, the effect of scinderin knockdown on cell proliferation was also investigated. The results of RTCA proliferation (Fig. 6A) demonstrated that there were no significant differences in the $\mathrm{CI}$ values between the $\mathrm{NC}$ and $\mathrm{KD}$ groups at the initial stage $(\mathrm{P}>0.05)$. Following $24 \mathrm{~h}$, however, the proliferation of SGC-7901 cells in the KD group was significantly lower than that in the $\mathrm{NC}$ group $(\mathrm{P}<0.01$ or $\mathrm{P}<0.001)$ and entered the stationary stage in advance. These data clearly indicated that scinderin knockdown effectively suppressed the proliferation of SGC-7901 gastric cancer cells.

The cell cycle distribution based on DNA content was examined by flow cytometric analysis. It was identified that there was a $1.03 \%$ increase in the number cells in $\mathrm{G} 2 / \mathrm{M}$-phase in the KD group compared with the NC group $(\mathrm{P}<0.01$, Fig. 6B). These results suggested that scinderin knockdown in SGC-7901 cells may arrest the cell cycle at G2/M phase.

\section{Discussion}

A study by Haifu Wu demonstrated that scinderin is the most differentially expressed gene in colon cancer patients with and without liver metastasis by gene-chip technology and qPCR assay (23). The study also identified, consistent with the results of the present study, that scinderin knockdown in colorectal cancer cell lines SW480 and DLD-1 evidently inhibited cell proliferation and migration. These results suggested that scinderin has important roles in the development and progression of cancer.

The present analyses of scinderin knockdown in highly metastatic SGC-7901 cells suggested that scinderin is associated with cell migration. It is well-established that the actin cytoskeleton contributes to maintaining the distinctive structures and functions of epithelial cells by mediating interactions with the cellular basement membranes and cell-cell contacts (24). Aberrance of the actin cytoskeleton is considered as one fundamental characteristic of the majority of malignant and metastatic cells. Studies performed in epithelial cells have demonstrated that gelsolin and villin, two proteins associated with the scinderin family, regulate cell migration by altering actin filament dynamics $(25,26)$. Scinderin is an important actin-capping, -severing and -nucleating protein. Since it can depolymerize actin filaments and bind actin monomers, it is hypothesized that the loss of scinderin in epithelial cells may increase the cellular levels of F-actin, thus modifying actin filament dynamics. Another role of scinderin may serve to regulate the proper distribution of F-actin filaments in cells.

Furthermore, scinderin may also regulate the EMT process to affect cell migration. The present study indicated that scinderin knockdown in SGC-7901 cells resulted in a high increase of E-cadherin and reduced the expression of N-cadherin. E-cadherin as an important $\mathrm{Ca}^{2+}$-dependent adhesion molecule has key roles in the cell adhesion of solid tissues. It is anchored to the actin cytoskeleton via $\alpha-, \gamma$ - and $\beta$-catenin, providing the physical structure for both cell-cell attachment and the recruitment of signaling complexes (27). Loss or inactivation of E-cadherin is generally regarded as the main trigger for the disruption of tight epithelial cell-cell contacts, causing migratory or invasive states of malignant cells. In several carcinoma types, a deficiency of E-cadherin expression is most commonly accompanied by the gain of $\mathrm{N}$-cadherin, which has been established as the cadherin switch in the EMT process. Although the functional implication of this cadherin switch for tumor progression has remained unknown, the overexpression of N-cadherin may be equally necessary and sufficient to overcome E-cadherin-mediated cell-cell adhesion and to promote therioma development. Of note, gelsolin has also been found to regulate EMT in human mammary epithelial cells. However, this is inconsistent with the present results that gelsolin knockdown by siRNA in MCF10A cells induces EMT, controlling E-cadherin and N-cadherin conversion via Snail (28).

Notably, the present study identified that the loss of scinderin reduced the expression of $\beta$-catenin protein, a key nuclear effector of canonical Wnt signaling in the nucleus. The Wnt/ $\beta$-catenin pathway is one of the fundamental signaling pathways controlling EMT, which may facilitate the expression of key transcriptional repressors that target E-cadherin, including Snail2, ZEB1 and Twist (29-31). Based on present knowledge, it is suggested that overexpression of scinderin may enhance free cytoplasmic levels of $\beta$-catenin by altering the actin cytoskeleton or other mechanisms. $\beta$-catenin, escaping cytoplasmic degradation, translocates into the cell nucleus and triggers transcription of Wnt-specific genes (32). 

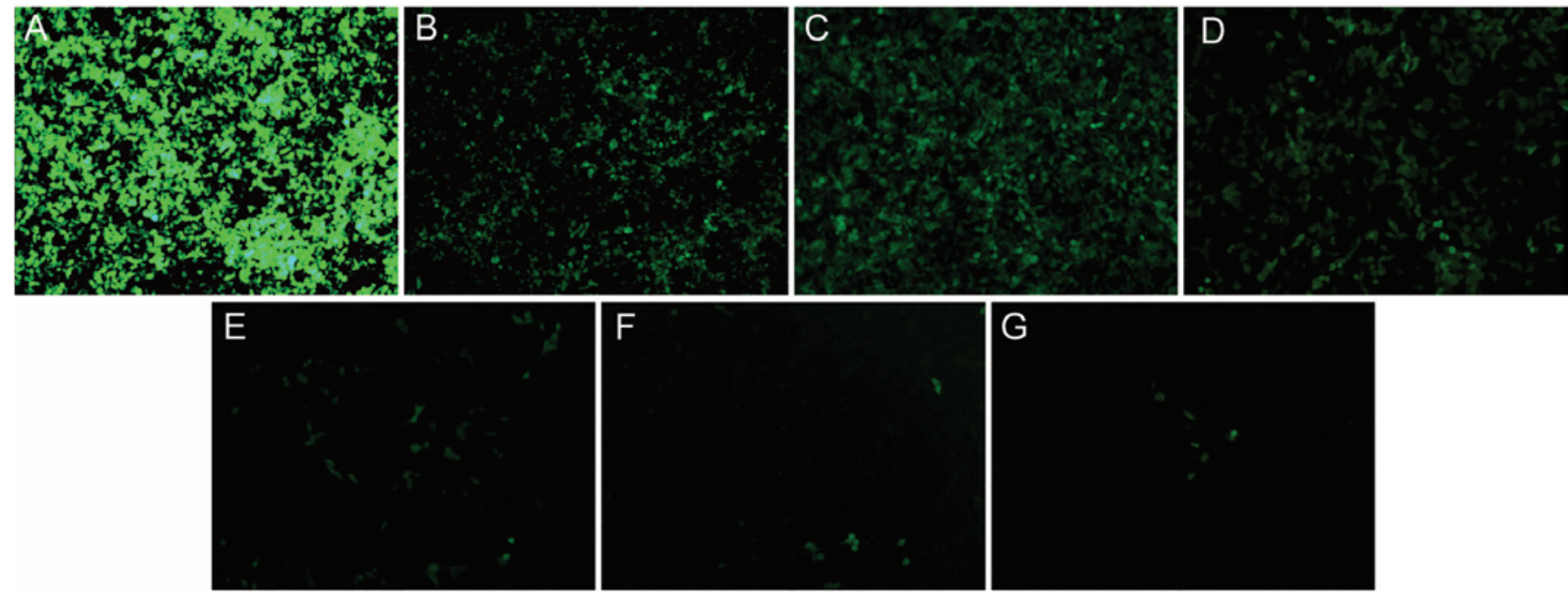

Figure 2. Fluorescence microscopic images of $293 \mathrm{~T}$ cells after $96 \mathrm{~h}$ of transfection with lentivirus (magnification, $\mathrm{x} 100$ ). (A-G) Volume of lentivirus is 10,1 , $10^{-1}, 10^{-2}, 10^{-3}, 10^{-4}, 10^{-5}$.

A

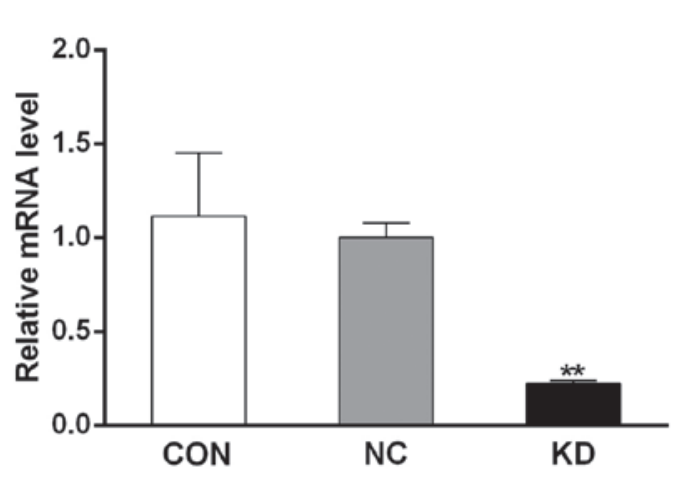

B

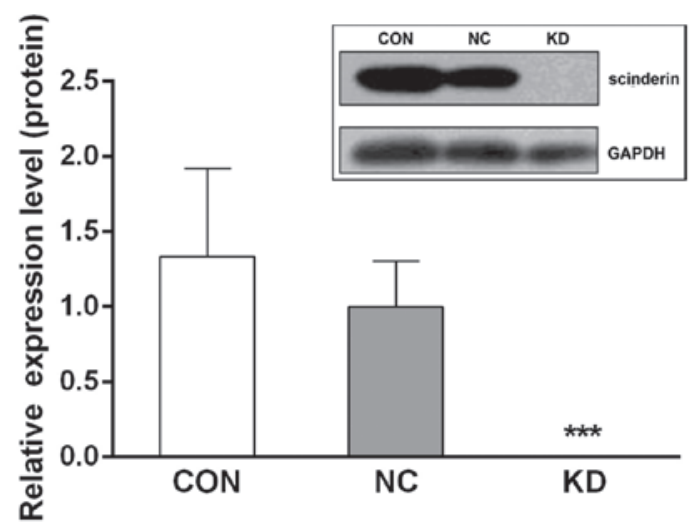

Figure 3. Expression of scinderin in SGC-7901 cells at mRNA and protein levels. (A) Quantitative reverse-transcription polymerase chain reaction analysis of scinderin mRNA levels. (B) Western blot analysis of scinderin protein levels. GAPDH was used as the loading control. Values are presented are the mean \pm standard deviation of three independent experiments ( ${ }^{* *} \mathrm{P}<0.01,{ }^{* * *} \mathrm{P}<0.001$ as compared with the CON group).CON, control group; NC, empty vector group; KD, experimental (scinderin-shRNA) group.
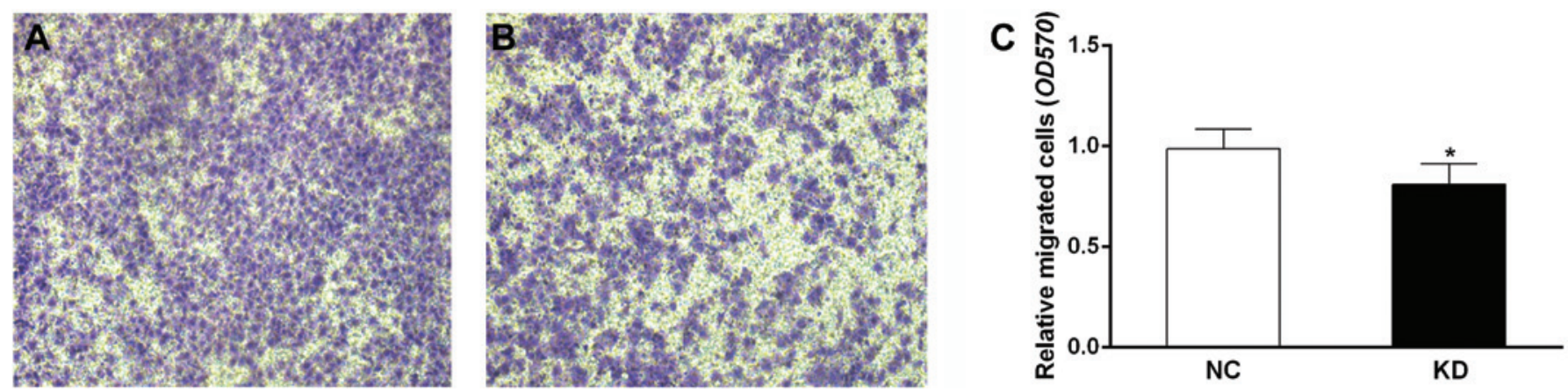

Figure 4. Effect of scinderin knockdown on cell migration analyzed by Transwell assay (magnification, x100). (A) NC group. (B) KD group. (C) Scinderin knockdown significantly reduced the migration of SGC-7901 cells. The results represent the average of three independent experiments. "P<0.05 as compared with NC group. NC, empty vector group; KD, experimental (scinderin-shRNA) group.

As a result, the expression of E-cadherin, a key hallmark of EMT, is highly aberrant in epithelial cells. Cadherins appear to directly affect the function of each other. N-cadherin, one of the mesenchymal cadherins, is highly expressed at the same time and facilitates the acquisition of a migratory phenotype.
Whether this conjecture is valid remains to be investigated in the future. The physiological function of scinderin is remains limited to actin filaments. Thus far, it has been confirmed that changes of megakaryoblastic cells brought about by scinderin expression are mediated through the activation 
A

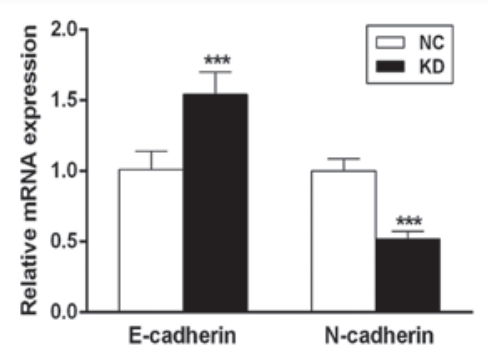

B

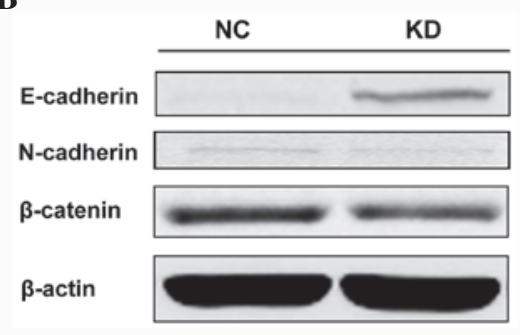

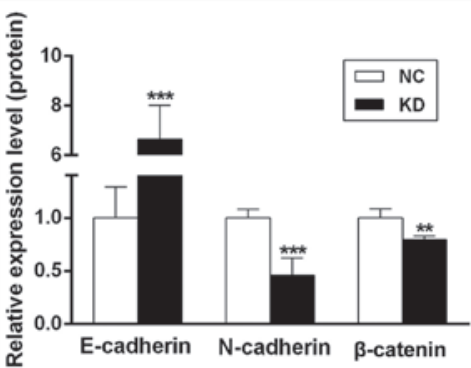

Figure 5. Expression of important EMT markers including E-cadherin, N-cadherin and $\beta$-catenin. (A) Quantitative reverse-transcription polymerase chain reaction analysis of E-cadherin and N-cadherin expression in SGC-7901 cells treated with scinderin knockdown. (B) Western blot analysis of E-cadherin, $\mathrm{N}$-cadherin and $\beta$-catenin expression. $\beta$-actin was used as a loading control. Values are presented as the mean \pm standard deviation of triplicate assays $\left({ }^{* * *} \mathrm{P}<0.01\right.$,

${ }^{* * *} \mathrm{P}<0.001$ as compared with NC group). EMT, epithelial-mesenchymal transition; NC, empty vector group; KD, experimental (scinderin-shRNA) group.
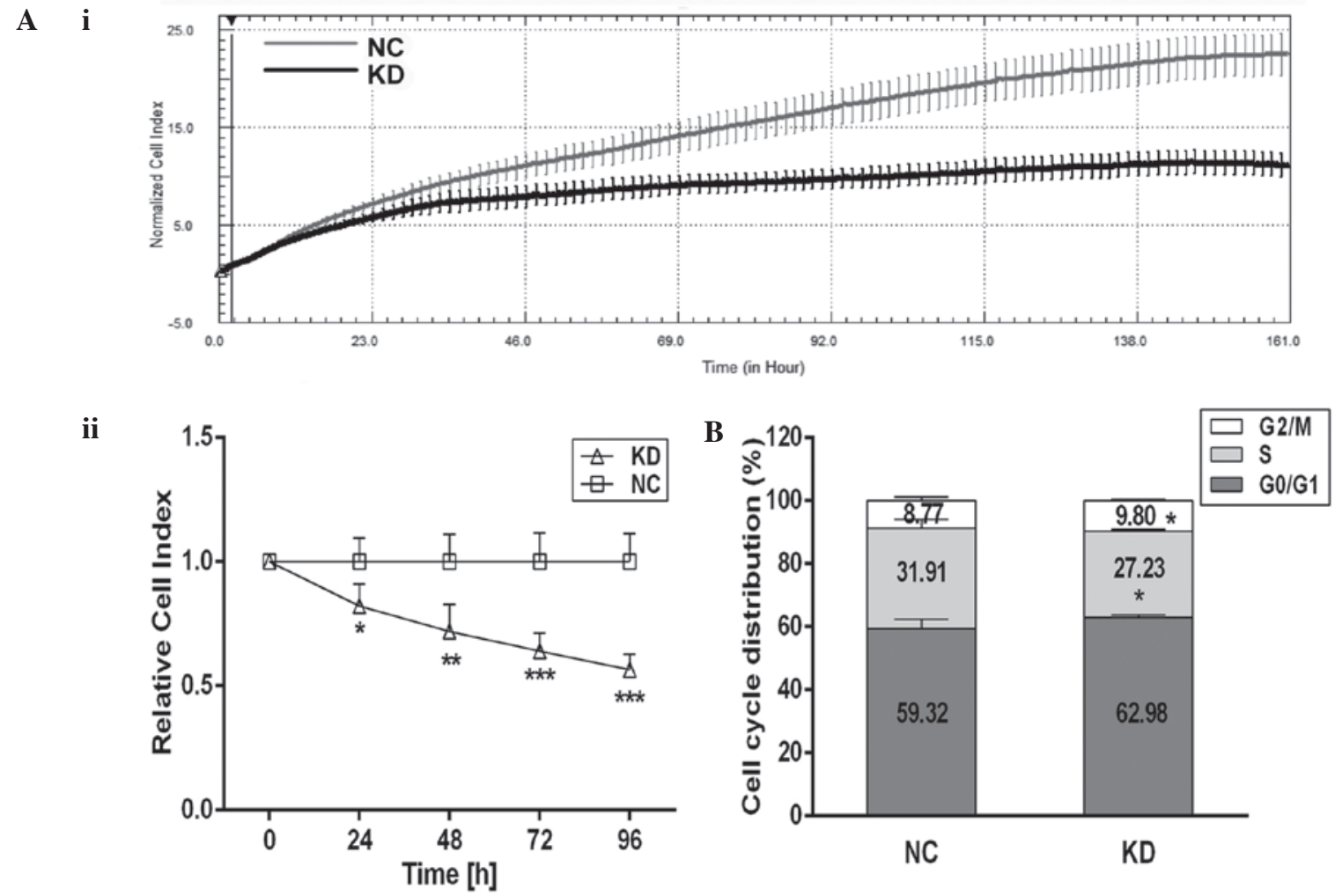

Figure 6. Effect of scinderin knockdown on cell proliferation and cell cycle distribution. (Ai) RTCA proliferation results and (ii) data analyses after 0, 24, 48, 72 and 96 h; scinderin knockdown significantly inhibited cell proliferation. (B) Stable transfected SGC-7901 cells were stained with propidium iodide and analyzed for cell-cycle distribution. All results represent the average of three independent experiments. ${ }^{*} \mathrm{P}<0.05,{ }^{* * *} \mathrm{P}<0.01$ and ${ }^{* * * *} \mathrm{P}<0.001$ compared with $\mathrm{NC}$ group. RTCA, real-time cellular analysis; NC, empty vector group; KD, experimental (scinderin-shRNA) group.

of rho family of guanosine triphosphatase/p21 activated kinase/mitogen-activated protein kinase kinase kinase. Mitogen-activated protein kinase kinase 4/c-Jun N-terminal kinase/c-jun, c-fos and Raf/mitogen-activated protein kinase kinase/extracellular signal-regulated kinase pathways, the only molecular mechanisms reported that scinderin participates in (10). However, the present study indicated that scinderin may act as a potential positive regulator in the EMT process of gastric cancer cells.

In addition, the present study identified that the cell cycle was arrested in G2/M phase (based on DNA content), which may lead to impairment of proliferation of SGC-7901 cells with low scinderin expression. However, a recent study showed that scinderin silencing reduced the proliferation and colony forma- tion of prostate cancer cell line PC3 by arresting the cell cycle at the $\mathrm{G} 0 / \mathrm{G} 1$ phase (33). The mRNA level of cell cycle-related molecules was also assessed by RT-qPCR analysis, indicating that scinderin silencing induces upregulation of $\mathrm{p} 21$ waf1/cip1 and p16 and downregulation of cyclin A2 in PC 3 cells. In view of the selectivity of gene expression in different tissues, these two studies perhaps do not appear to be consistent: SGC-7901 cells are derived from lymph node metastasis of gastric cancer, and PC 3 cells are from bone metastasis in prostate cancer, both of which are in different microenvironment for tumor growth and survival. Flow cytometry is unnable to distinguish between $\mathrm{G} 2$ and M phase of the cell cycle, which may explain why the present study demonstrated that scinderin knockdown significantly suppressed the proliferation of SGC-7901 cells 
but the changes in cell-cycle distribution were not evident. As is well-established, microfilaments have a number of important roles in the telophase of cell division. The function of scinderin in regulating actin dynamics has already been clearly demonstrated. It was therefore suggested that scinderin may effect cell proliferation by regulating F-actin; however, further studies are required to confirm this hypothesis.

The metastases of carcinomas are formed following a complex succession of cell-biological events including local invasion and metastatic colonization (34). As mentioned previously, scinderin expression is low or absent in the human adult stomach. The results of the present study suggested that: i) scinderin knockdown is able to reverse EMT process and effectively prevent migration of highly metastatic SGC-7901 cells, which also can be understood to weaken the local invasion in primary gastric cancer; ii) scinderin knockdown is able to inhibit the proliferation of SGC-7901 cells, greatly impairing the proliferation of cancer cells at metastatic sites and the formation of metastatic colonization. If human gastric cancer tissues provide similar results to those of SGC-7901 cells highly expressing scinderin, which is closely associated with carcinoma metastasis, scinderin may be a useful prognostic biomarker to distinguish the progression of gastric cancer and guide personalized therapy for patients.

In conclusion, the present study demonstrated that suppression of scinderin impaired the proliferation and migration of gastric cancer SGC-7901 cells and attenuated their EMT process. Therefore, scinderin may be a potential target for tumor EMT and therapy against gastric cancer.

\section{Acknowledgements}

The present study was supported by the Natural Science Foundation of Zhejiang Province (grant no. Y2110961) and Natural Science Foundation of Ningbo (grant no. 2011A610051).

\section{References}

1. Marcu MG,Zhang L, Elzagallaai A and Trifaró JM: Localization by segmental deletion analysis and functional characterization of a third actin-binding site in domain 5 of scinderin. J Biol Chem 273: 3661-3668, 1998.

2. Lejen T, Pene TD, Rosé SD and Trifaró JM: The role of different Scinderin domains in the control of F-actin cytoskeleton during exocytosis. Ann NY Acad Sci 971: 248-250, 2002.

3. Dumitrescu Pene T, Rosé SD, Lejen T, Marcu MG and Trifaró JM: Expression of various scinderin domains in chromaffin cells indicates that this protein acts as a molecular switch in the control of actin filament dynamics and exocytosis. J Neurochem 92: 780-789, 2005.

4. Chumnarnsilpa S, Lee ML, Nag S, et al: The crystal structure of the C-terminus of adseverin reveals the actin-binding interface. Proc Natl Acad Sci USA 106: 13719-13724, 2009.

5. Lueck A, Brown D and Kwiatkowski DJ: The actin-binding proteins adseverin and gelsolin are both highly expressed but differentially localized in kidney and intestine. J Cell Sci 111: 3633-3643, 1998.

6. Trifaró JM, Vitale ML and Rodríguez Del Castillo A: Scinderin and chromaffin cell actin network dynamics during neurotransmitter release. J Physiol Paris 87: 89-106, 1993.

7. Trifaró JM, Rosé SD and Marcu MG: Scinderin, a $\mathrm{Ca}^{2+}$-dependent actin filament severing protein that controls cortical actin network dynamics during secretion. Neurochem Res 25: 133-144, 2000

8. Trifaró JM, Gasman S and Gutiérrez LM: Cytoskeletal control of vesicle transport and exocytosis in chromaffin cells. Acta Physiol (Oxf) 192: 165-172, 2008.
9. Ehre C, Rossi AH, Abdullah LH, et al: Barrier role of actin filaments in regulated mucin secretion from airway goblet cells. Am J Physiol Cell Physiol 288: C46-C56, 2005.

10. Zunino R, Li Q, Rosé SD, et al: Expression of scinderin in megakaryoblastic leukemia cells induces differentiation, maturation, and apoptosis with release of plateletlike particles and inhibits proliferation and tumorigenesis. Blood 98: 2210-2219, 2001.

11. Bush WS, McCauley JL, DeJager PL, et al: A knowledge-driven interaction analysis reveals potential neurodegenerative mechanism of multiple sclerosis susceptibility. Genes Immun 12: 335-340, 2011.

12. Abouzahr S, Bismuth G, Gaudin C, et al: Identification of target actin content and polymerization status as a mechanism of tumor resistance after cytolytic T lymphocyte pressure. Proc Natl Acad Sci USA 103: 1428-1433, 2006.

13. Miura N, Takemori N, Kikugawa T, et al: Adseverin: a novel cisplatin-resistant marker in the human bladder cancer cell line HT1376 identified by quantitative proteomic analysis. Mol Oncol 6: 311-322, 2012.

14. Zavadil J, Haley J, Kalluri R, Muthuswamy SK and Thompson E: Epithelial-mesenchymal transition. Cancer Res 68: 9574-9577, 2008.

15. Kalluri R and Weinberg RA: The basics of epithelial-mesenchymal transition. J Clin Invest 119: 1420-1428, 2009.

16. Scanlon CS, Van Tubergen EA, Inglehart RC and D'Silva NJ: Biomarkers of epithelial-mesenchymal transition in squamous cell carcinoma. J Dent Res 92: 114-121, 2013.

17. Turley EA, Veiseh M, Radisky DC and Bissell MJ: Mechanisms of disease: epithelial-mesenchymal transition-does cellular plasticity fuel neoplastic progression. Nat Clin Pract Oncol 5: 280-290, 2008.

18. De Craene B and Berx G: Regulatory networks defining EMT during cancer initiation and progression. Nat Rev Cancer 13: 97-110, 2013.

19. Zheng $\mathrm{H}$ and Kang Y: Multilayer control of the EMT master regulators. Oncogene 33: 1755-1763: 2013.

20. Iorio MV and Croce CM: MicroRNA dysregulation in cancer: diagnostics, monitoring and therapeutics. A comprehensive review. EMBO Mol Med 4: 143-159, 2012.

21. Beltran M, Puig 1, Peña C, et al: A natural antisense transcript regulates Zeb2/Sip1 gene expression during Snail1-induced epithelial-mesenchymal transition. Genes Dev 22: 756-769, 2008.

22. Luo M, Li Z, Wang W, et al: Long non-coding RNA H19 increases bladder cancer metastasis by associating with EZH2 and inhibiting E-cadherin expression. Cancer Lett 333: 213-221, 2013.

23. Haifu Wu: Correlated function study of scinderin gene in liver metastasis of colorectal cancer (unpublished $\mathrm{PhD}$ thesis). Fudan University, 2010.

24. Khurana S and George SP: Regulation of cell structure and function by actin-binding proteins: villin's perspective. FEBS Lett 582: 2128-2139, 2008.

25. Tomar A, Wang Y, Kumar N, et al: Regulation of cell motility by tyrosine phosphorylated villin. Mol Biol Cell 15: 4807-4817, 2004.

26. Li GH, Arora PD, Chen Y, McCulloch CA and Liu P: Multifunctional roles of gelsolin in health and diseases. Med Res Rev 32: 999-1025, 2012.

27. Valenta T, Hausmann G and Basler K: The many faces and functions of $\beta$-catenin. EMBO J 31: 2714-2736, 2012.

28. Tanaka H, Shirkoohi R, Nakagawa K, et al: siRNA gelsolin knockdown induces epithelial-mesenchymal transition with a cadherin switch in human mammary epithelial cells. Int J Cancer 118: 1680-1691, 2006.

29. Conacci-Sorrell M,Simcha I, Ben-Yedidia T, et al: Autoregulation of E-cadherin expression by cadherin-cadherin interactions: the roles of beta-catenin signaling, Slug, and MAPK. J Cell Biol 163: 847-857, 2003.

30. Onder TT, Gupta PB, Mani SA, et al: Loss of E-cadherin promotes metastasis via multiple downstream transcriptional pathways. Cancer Res 68: 3645-3654, 2008.

31. Gradl D, Kühl M and Wedlich D: The Wnt/Wg signal transducer beta-catenin controls fibronectin expression. Mol Cell Biol 19: 5576-5587, 1999.

32. Anastas JN and Moon RT: WNT signalling pathways as therapeutic targets in cancer. Nat Rev Cancer 13: 11-26, 2013.

33. Wang D, Sun SQ, Yu YH, et al: Suppression of SCIN inhibits human prostate cancer cell proliferation and induces G0/G1 phase arrest. Int J Oncol 44: 161-166, 2014.

34. Valastyan S and Weinberg RA: Tumor metastasis: molecular insights and evolving paradigms. Cell 147: 275-292, 2011. 